\title{
Microcrystalline hydroxyapatite compound in prevention of bone loss in corticosteroid-treated patients with chronic active hepatitis
}

\author{
A. Stellon ${ }^{1}$, A. Davies ${ }^{1}$, A. Webb ${ }^{2}$ and R. Williams ${ }^{1}$ \\ ${ }^{1}$ Liver Unit and Dept. of Radiology, King's College Hospital, London S.E.5, and ${ }^{2}$ Dept. of Histopathology, \\ St. Thomas' Hospital, London S.E.1, UK.
}

\begin{abstract}
Summary: To determine whether microcrystalline hydroxyapatite compound (MCHC) could reduce bone loss or its consequences in patients with chronic active hepatitis (CAH) on corticosteroid therapy, a controlled trial was conducted in 36 such patients over a period of 2 years. Both skeletal symptoms (back pain) and fractures were uncommon during the trial period but both showed non-significant differences in favour of the MCHC group and biochemical investigations were suggestive of a reduction in parathyroid over-activity. Continued reduction in bone mineral content of the radius (photon absorptiometry) was halted in those receiving MCHC and iliac crest bone biopsy showed a non-significant increase in trabecular bone volume. The fall in iliac crest cortical plate thickness was significantly less $(P<0.025)$ in the MCHC group and the results overall were consistent with a beneficial effect from MCHC in corticosteroid-induced osteoporosis.
\end{abstract}

\section{Introduction}

Corticosteroid treatment of auto-immune chronic active hepatitis causes bone loss (Kato et al., 1982; Stellon et al., 1984) both by reducing bone formation and by increasing parathyroid-mediated resorption secondary to calcium malabsorption (Jee \& Clark, 1981). Treatment or prevention of this complication, as with osteoporosis in general, seeks to both decrease bone resorption and increase bone formation. The latter is the more difficult to achieve and the reported usefulness of sodium fluoride is limited by a high incidence of side effects (Briancon \& Meunier, 1981; Riggs et al., 1981).

To decrease bone resorption oestrogens, anabolic steroids, calcitonin, calcium supplementation or a combination of these have been used (Nagant de Deuxchaisnes, 1983), although contra-indications exist for several of these in patients with chronic liver disease. Calcium suppplementation is virtually without adverse effects and microcrystalline hydroxyapatite compound (MCHC; 'Ossopan') offers calcium in a form which is more bioavailable than in other soluble calcium preparations (Windsor et al., 1973; Dent \& Davies, 1980; Durance et al., 1973) and its much lower sodium content is of value in patients with chronic liver disease (Epstein et al., 1982). In the present report we describe a controlled clinical trial of

Correspondence: R. Williams, M.D., F.R.C.P.

Accepted: 14 May 1985
MCHC in patients with auto-immune chronic active hepatitis on long-term corticosteroid therapy.

\section{Materials and methods}

Thirty-six patients ( 34 female), aged 20 to $66 \mathrm{y}$, fulfilled internationally agreed criteria for autoimmune CAH (Review by International Group, 1977). At the time of entry, biochemical and histological remission of the disease had been achieved in all patients. All patients had received prednisolone for a minimum period of $1 \mathrm{y}$ before the trial, the maintenance dose at the time of entry ranging from 5 to $12.5 \mathrm{mg} / \mathrm{d}$. Thirty-one patients were also receiving azathioprine $(50-100 \mathrm{mg} / \mathrm{d})$ and 6 spironolactone for fluid retention. None of the patients had received previous treatment with vitamin $D$ or calcium supplements and none showed clinical evidence of steatorrhoea. Informed consent was obtained from all patients.

\section{Study design}

Patients were randomized to receive either $\mathrm{MCHC}$ powder $8 \mathrm{~g} / \mathrm{d}$ or no calcium supplementation. Patients finding the MCHC powder unpalatable were allowed to change to an equivalent daily dose of tablets. At entry to the study all patients were interviewed by an 
experienced dietician, to obtain an estimate of the daily intake of calcium and vitamin $D$, and also completed a questionnaire relating to bone pains, analgesic consumption and past fractures. Patients underwent biochemical investigations, radiology of the hand and thoraco-lumbar spine and single photon absorptiometry, at trial entry and at yearly intervals for $2 y$. A radio-calcium absorption test and an iliac crest bone biopsy were perfomed at entry with the latter being repeated at the end of the 2 year study.

\section{Biochemistry}

Venous blood samples were collected, without stasis, together with a urine sample after a $12 \mathrm{~h}$ overnight fast. Serum concentrations of calcium, phosphorus and creatinine, urinary calcium and creatinine levels and fasting calcium:creatinine ratios were obtained by standard methods. Plasma 25-hydroxyvitamin D was measured by a competitive protein-binding assay after extraction and chromatography. The fractional absorption of calcium (percentage absorption of administered dose), was measured by the double isotope method (Wooton \& Reeve, 1980) after a 12 h overnight fast. The isotopes used were ${ }^{47}$ calcium chloride $(10-15 \mu \mathrm{Ci})$ orally and ${ }^{85}$ strontium chloride $(2 \mu \mathrm{Ci})$ intravenously given simultaneously in which blood is sampled serially over the following $6 \mathrm{~h}$ and the results analysed using a computer programme written in Fortran IV.

\section{Radiology}

Serial postero-anterior radiographs of the right hand were obtained and from these the cortical thickness (MCT) and ratio of cortical area to total area (CA:TA) were determined (Wooton \& Reeve, 1980) for the 2nd metacarpal bone (coefficient of variation $1.1 \%$ ). Radiographs of the thoraco-lumbar spine were assessed for the presence of vertebral body changes (Nordin et al., 1976). Radiographs were evaluated by a single observer over a period of 1 week.

\section{Photon absorptiometry}

Bone mineral content (BMC) of the radius was determined by single photon absorption, employing a Gambro Bone Mineral Detector with a 241 Americium Radiation Source, $8 \mathrm{~cm}$ from the distal radial styloid process in both arms. The soft tissue baseline count was determined at a point between the radius and ulna which gave the maximum counts so as to eliminate corrections for the degree of fat in such individuals. Results were calculated as the mean of four scans performed at each site without repositioning and expressed in $\mathrm{g} / \mathrm{cm}$ (coefficient of variation $2.7 \%)$.

\section{Bone histomorphometry}

Two full thickness transiliac crest bone biopsies were obtained, $2 \mathrm{~cm}$ below and posterior to the anterior superior iliac spine, using an $8 \mathrm{~mm}$ modified Bordier? trephine to reduce the effect of inter-sample variability. The biopsy at conclusion of the trial was performed on the opposite iliac crest to the previous biopsy. Non-consecutive undecalcified $7 \mu \mathrm{m}$ thick $\varrho$ sections were stained by the von Kossa techniqueis using a van Giesen counterstain. The total trabecular bone volume (TBV), mineralized and unmineralized; expressed as a percentage of the total medullary $\vec{\omega}$ volume including both bone and marrow spaces, was measured using an eyepiece graticule on a minimum ofo 100 fields from four or more sections. The cortical? plate thickness (CPT), the mean width of both innerand outer cortical plates, was measured using an eyepiece micrometer as previously described (Vedi et. al., 1982) on three von Kossa stained sections. Allç sections were quantitated by one observer blind of the $\mathrm{e}_{-}^{-}$ patients' therapy.

\section{Statistical analysis}

All measurements consisted of continuous variables $\stackrel{\mathbb{D}}{3}$ whose distribution was such as to justify the use gfo parametric statistical methods. Within-group changs and between-group differences were tested for sus-o nificance using two- and three-way analyses of ${ }^{\mathrm{r}}$ variance, as appropriate. Two-tailed probability levelso were determined in all cases and null hypotheses rejected at levels less than 0.05 . Since the clinicalo situation dictated that a parallel group design had too be employed, the statistical power of tests performed $\stackrel{\mathbb{Q}}{\varrho}$ on changes during therapy (within-patient compar $-\overrightarrow{\vec{O}}$ isons) is inevitably much greater than the power of 3 tests between the groups (between-patient comparisons). Between-group effects have been assessed as? the group $\times$ time interaction term, thereby effectivelyo comparing the changes during the trial in the two groups.

\section{Results}

The control and MCHC-treated groups were com-D parable with respect to demographic factors, presence of cirrhosis, the mean daily dose and dose-durationn (mean dose $x$ duration of therapy) of corticosteroid therapy and the dietary intake of calcium and vitamin D (Table I). Two patients, one in each group, werew withdrawn from the trial at their own request, in both instances during the first 6 months. Of the remainingo 17 patients in the MCHC group, 5 disliked the taste of the powder and 3 of these changed to tablets.

At entry 3 patients had skeletal symptoms (mild 
Table I Comparison of demographic and clinical variables and dietary data in MCHC-treated and control chronic active hepatitis patients

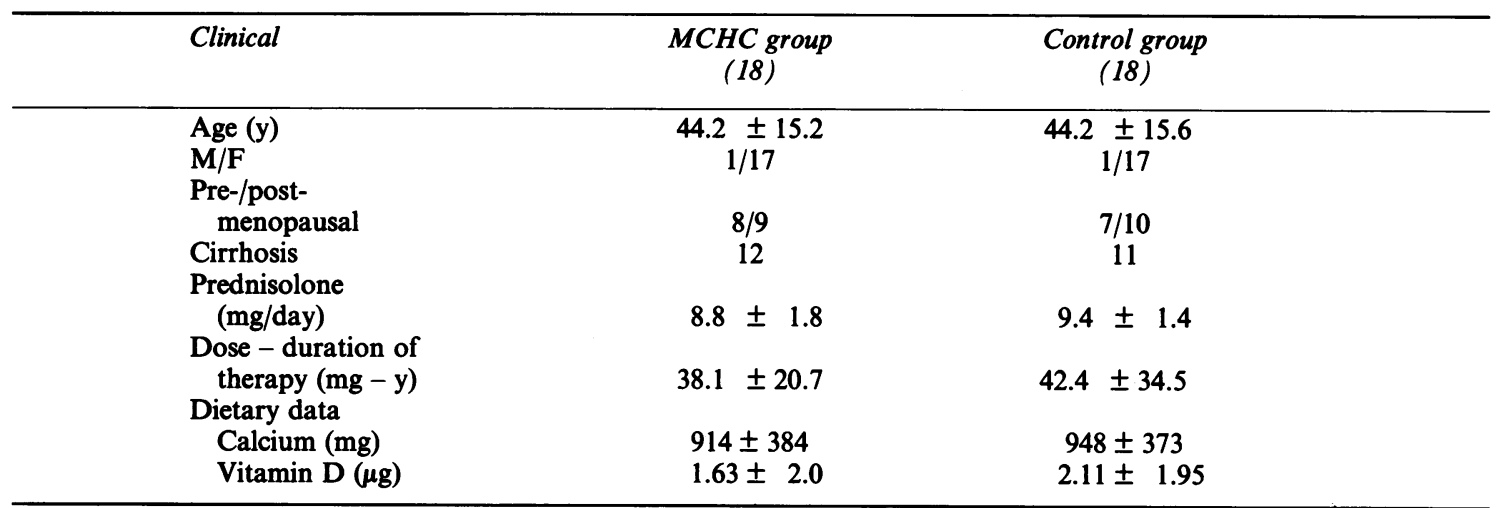

Results are expressed as mean \pm s.d.

lower lumbar pain). Two of these subsequently received MCHC; the pain disappeared within 6 months in one and remained throughout the trial in the other as it did in the third symptomatic patient in the control group. Of the remaining initially asymptomatic patients, two (both in control group) developed back pain (one thoracic, one lumbar) during the 2 year trial period; no previously asymptomatic patients developed skeletal pain in the MCHC group.

The mean serum calcium and phosphate levels, fasting urinary calcium: creatinine ratio, and mean 25 hydroxyvitamin D levels as well as the fractional absorption of calcium were comparable in the two groups at entry to the study (Table II). A nonsignificant decrease in serum calcium and fasting calcium:creatinine ratio occurred in the MCHC treated group, compared to controls, at the end of the 2-year period (Table II).

One patient had radiological evidence of a vertebral fracture (a single wedged vertebra) at entry and during the subsequent 2 y three patients in the control group, but none in the MCHC group, developed wedged vertebrae, but this failed to reach statistical significance. One patient in each group suffered a Colles' fracture during the trial ( 1.5 and $2 \mathrm{y}$ after entry); in the MCHC treated patient this was seen in a radius that had previously fractured.

There were significant and similar reductions during the trial in both groups in both cortical thickness $(P<0.025$ time effect $)$ and CA:TA ratio $(P<0.01$ time effect) of the 2nd metacarpal (Figure 1). Bone mineral content of the radius, as assessed by absorptiometry, decreased significantly during the trial in the controls $(P<0.005,2$-way Anovar) but not in the MCHC treated patients (Figure 1). The difference in bone mineral loss between the two groups just failed to reach statistical significance (3-way Anovar).

The TBV figures from the bone biopsies were not significantly different in the two groups; 5 patients (4 in MCHC group, 1 control), had initial figures less

Table II Clinical data in MCHC-treated and control CAH patients at entry and on completion of the $2 \mathrm{y}$ study period

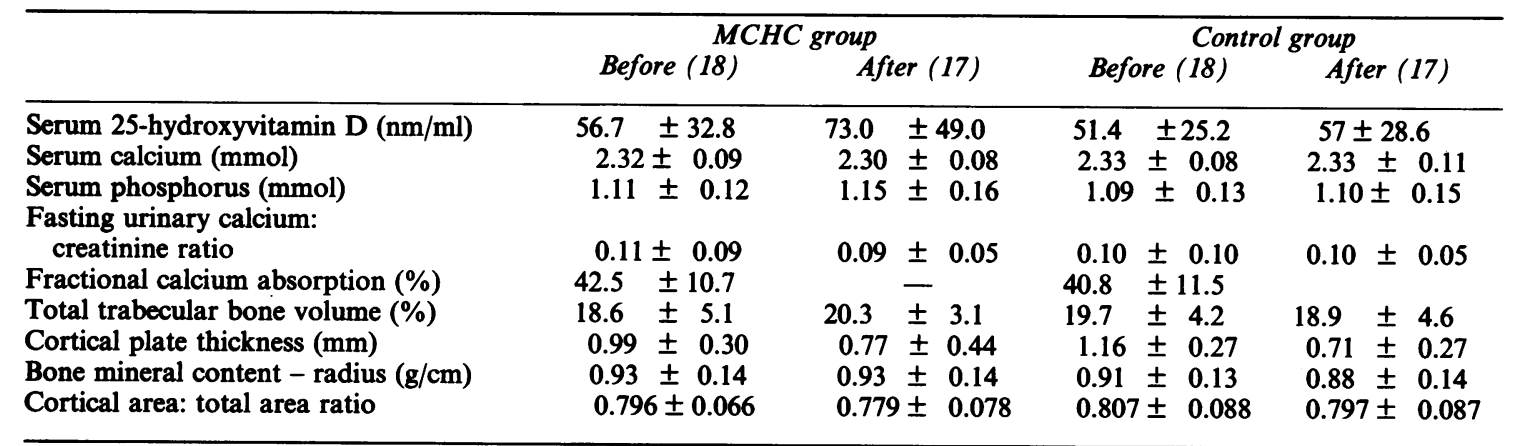

Results are expressed as mean \pm s.d. 


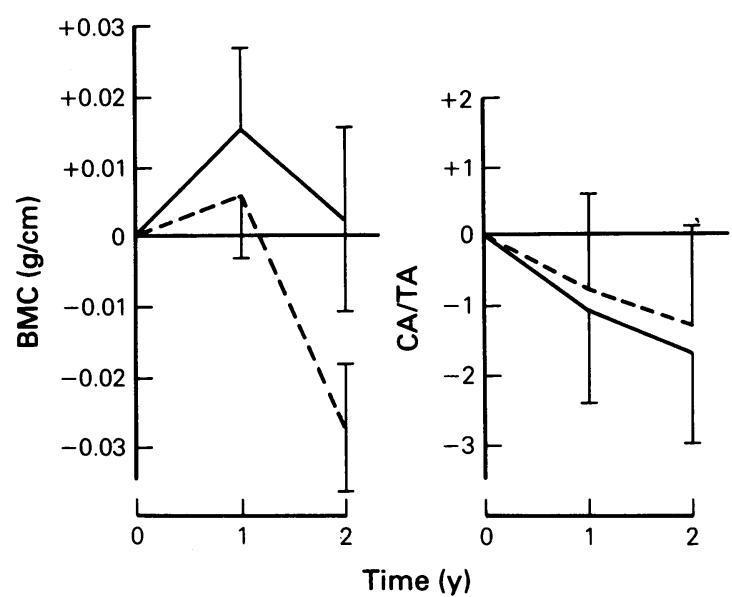

Figure 1 The mean changes from baseline of BMC of the radius and CA/TA ratio of the 2 nd metacarpal in the MCHC-treated (- $)$ and controls (-...-) during the $2 \mathrm{y}$ study period. The bars indicate the standard error of the mean.

than $14 \%$. In the MCHC group, there was a (statistically non-significant) rise in TBV during the $2 \mathrm{y}$ trial period, contrasting with a small (again non-significant) fall in the control group (Figure 2). Despite the difference in direction of change in the two groups, the difference between groups with respect to TBV changes did not reach statistical significance, due mainly to the large between-patient variability of results with the number of patients in the two groups. Cortical plate thickness assessed by bone biopsy, fell significantly during the trial $(P<0.0001$ time effect $)$ but the fall was significantly less $(P<0.025,3$-way Anovar) in the MCHC group than in the control group (Figure 2).

\section{Discussion}

The present results show that MCHC alone may be effective in reducing bone loss and possibly also in preventing fractures in patients with CAH on long term corticosteroid therapy. Symptomatically, $\mathrm{MCHC}$ was associated with resolution of back pain in one of two patients in the treated group whereas two control patients developed such pain during the period of observation. There was also a smaller number of fractures in the MCHC group although this failed to reach statistical significance. The single instance (Colles' fracture) that occurred in the MCHC group may have been related to the previous fracture in the same bone.

The possible prophylactic effect of MCHC against vertebral fractures is supported by the bone biopsy data. Five patients had a trabecular bone volume

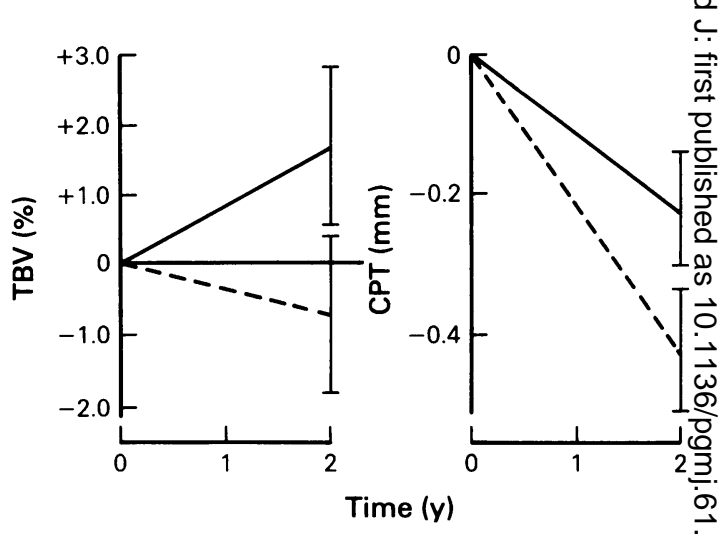

Figure 2 The mean changes from baseline of TBV and $\stackrel{\sim}{\oplus}$ CPT of the iliac crest in MCHC-treated (-) and controls (-...-) during the 2 y study period. The bars $\underset{ }{ }$ indicate the standard error of the mean.

below the 'vertebral fracture threshold' of $11 \% \pm 3 \%$ as proposed by Meunier et al., 1979, and none of the four treated with MCHC developed vertebral frac tures during the study. In contrast, vertebral wedging developed in three controls with TBV values $>16 \%$ age the start of the trial and in each instance the value ell subsequently to below fracture threshold leveb Although mean TBV values increased over the 2 period with MCHC treatment but fell slightly in the control group the difference just failed to reach statistical significance.

The significant decrease in the amount of corticat plate thinning (in iliac crest bone biopsies) in the treated group implies that MCHC prevents or de creases endosteal resorption. Suppression of corticos? teroid-induced secondary hyperparathyroidism is likely mechanism and the small reduction in urinary. calcium:creatinine ratio during MCHC therapy is alse consistent with an inhibition of bone resorption.

The importance of bone biopsy data, particularl cortical plate thickness, in assessing modifications to the risk of osteoporotic fractures was shown by Meunier in a recent study of idiopathic femoral nec fracture cases (Meunier et al., 1983). In a detailes. study from this Unit (Stellon et al., 1984) over 30\% of corticosteroid-treated patients with auto-immune CAH had significant cortical plate thinning with consequently high risk of developing femoral neck fractures at an early age. It is surprising that the beneficial effects of MCHC on iliac crest bone histoo? morphology were not reflected by similar finding $\Phi$ with the radiological metacarpal measurements. Pos ${ }^{+}$ sibly this relates to the much higher rate of turnove 
$(60 \% / y)$ and volume/surface ratio of trabecular bone in the iliac crest than that of cortical bone (turnover, 3\%/year; Parfitt, 1982), with changes being seen at a much earlier time at the former site. However, a differential effect (Nordin et al., 1980) of MCHC treatment on bones at different sites cannot be excluded.

Despite the low turnover of cortical bone, MCHC appeared to reduce bone mineral loss of the radius and, of the several possible mechanisms, a reduction in endosteal resorption and/or a reversal of cortical porosity is the most likely, the latter being a significant contributor to bone loss in long bones (Parfitt, 1980). Although radial bone mineral content or bone mineral content/width measurements do not provide prognostic information as to likely occurrence of Colles' fractures (Horsman et al., 1980; Nilsson \& Westlin, 1974), the present findings do provide further evidence that $\mathrm{MCHC}$ is effective in reducing bone loss in both axial and appendicular skeleton.

The results of the present study complement those of investigators in other disorders with likely corticosteroid-induced osteoporosis. Thus, in rheumatoid arthritis, Nilson et al. (1978) showed that MCHC

\section{References}

BRIANCON, D. \& MEUNIER, P.J. (1981). Treatment of osteoporosis with fluoride, calcium and vitamin D. Orthopaedic Clinics of North America, 12, 629.

DENT, C.E. \& DAVIES, I.J. (1980). Calcium metabolism in bone disease: effects of treatment with microcrystalline hydroxyapatite compound and dihydrotachysterol. Journal of the Royal Society of Medicine, 73, 780.

DURANCE, R.A., PARSONS, V., ATKINS. C.J.. HAMILTON, E.B.D. \& DAVIES, C. (1973). Treatment of osteoporotic patients. A trial of calcium supplements (Ossopan) and ashed bone. Clinical Trials Journal, 10, 67.

EPSTEIN, O., KATO, Y., DICK, R. \& SHERLOCK, S. (1982). Vitamin-D, hydroxyapatite, and calcium gluconate in treatment of cortical bone thinning in post-menopausal women with primary biliary cirrhosis. American Journal of Clinical Nutrition, 36, 426.

GALASKO, C.S., RUSSELL, S. \& RUSHTON, S. (1984). Microcrystalline hydroxyapatite in patients with Colles' fractures. In Osteoporosis, Christiansen, C., Arnaud, C.D., Nordin, B.E.C., Parfitt, A.M., Peck, W.A. \& Riggs, B.L. (eds). p.663. Glostrup Hospital, Denmark. Stiftsbogtrykkin: Aalborg.

HORSMAN, A., NORDIN, B.E.C., AARON, J. \& MARSHALL, D.H. (1980). Cortical and trabecular osteoporosis and their relation to fractures in the elderly. In Osteoporosis: Recent Advances in Pathogenesis and Treatment. De Luca, H.F., Frost, H.M., Jee, W.S., Johnston, C.C. \& Parfitt, A.M. (eds). p. 175. University Park Press: Baltimore.

JEE, W.S. \& CLARK, I. (1981). Glucocorticoid-induced osteoporosis. Osteoporosis: Recent Advances in Pathogenesis and Treatment. De Luca, H.F., Frost, H.M., Jee, W.S. Johnston, C.C. \& Parfitt, A.M. (eds). p 331. University reduced losses of both radial bone and vertebral stem height together with reduction in back pain, and in chronic respiratory diseases, Pines et al. 1984) found MCHC both reduced metacarpal bone loss and produced marked improvements in symptomatology of their patients, although the trial lacked an adequate control group. The combination of MCHC (but not sodium calcium gluconate) and vitamin $\mathrm{D}$ resulted in an increase of cortical bone thickness during a 14month period of observation in patients with primary biliary cirrhosis (Epstein et al., 1982) compared to the group on vitamin D alone, and most recently, Galasko et al. (1984) have demonstrated beneficial effects on cortical bone thickness in patients with severe idiopathic osteoporosis. Our results suggest that MCHC treatment may be effective in preventing corticosteroid-induced bone loss in patients with autoimmune CAH.

\section{Acknowledgements}

We thank Dr R. Kohn of Advisory Services (Clinical \& General) Limited, London, for organisational support.

Park Press: Baltimore.

KATO, Y., EPSTEIN, O., DICK, R. \& SHERLOCK, S. (1982). Radiological patterns of cortical bone modelling in women with chronic liver disease. Clinical Radiology, 33, 313.

MEUNIER, P.J., COURPRON, P., EDOUARD, C., ALEXANDRE, C., BRESSOT, C., LIPS, P. \& BOYCE, B.F. (1979). Bone histomorphometry in osteoporosis. In . Osteoporosis II. Barzel, V.S. (ed). p. 24, Grune and Stratton: New York. MEUNIER, P.J., BRIANCON, D., SELLAMI, S., EDOUARD, C., CHARAISSENX, P. \& ARLOT, M. (1983). Dynamic bone histomorphometry in primary osteoporosis. Osteoporosis: A Multidisciplinary Problem. Dixon, A.St.J., Russell, R.G.G. \& Stamp, T.C.B. (eds). p. 67. Academic Press: London and New York.

NAGANT DE DEUXCHAISNES, C. (1983). The pathogenesis and treatment of involutional osteoporosis. In Osteoporosis: A Multidisciplinary Problem. Dixon, A.St. J., Russell, R.G.G. \& Stamp, T.C.B. (eds). Academic Press, Grune and Stratton: London and New York.

NILSEN, K.H., JAYSON, M.I.V. \& DIXON, A.St.J. (1978). Microcrystalline calcium hydroxyapatite compound (Ossopan) in the prevention of osteoporosis due to corticosteroid therapy. Current Medical Research and Opinion, 8, 734.

NILSSON, B.O.\& WESTLIN, N.E. (1974). The bone mineral content in the forearm of women with Colles' fracture. Acta Orthopedica Scandinavica, 45, 836.

NORDIN, B.E.C., HORSMAN, A. \& AARON, J. (1976). Diagnostic procedures. In Calcium, Phosphate and Magnesium Metabolism. Nordin, B.E.C. (ed). p. 469. Churchill Livingstone: Edinburgh.

NORDIN, B.E.C., HORSMAN, A., CRILLY, R.G., MARSHALL, 
D.H. \& SIMPSON, M. (1980). Treatment of spinal osteoporosis in post-menopausal women. British Medical Journal, i, 451.

PARFITT, A.M. (1980). Morphologic basis of bone mineral measurements: transient and steady state effects of treatment in osteoporosis. Mineral Electrolyte Metabolism, 4, 273.

PARFITT, AM. (1982). The physiologic and clinical significance of bone histomorphometric data. Bone Histomorphometry Techniques and Interpretation. Recker, $\mathbf{R}$. (ed). p. 143. CRC Press: Bora, Ranton, Florida.

PINES, A., RAAFAT, H., LYNN, A.H. \& WHITTINGTON, J. (1984). Clinical trial of microcrystalline hydroxyapatite compound (Ossopan) in the prevention of osteoporosis due to corticosteroid therapy. Current Medical Research and Opinion, 8, 734.

REVIEW BY INTERNATIONAL GROUP (1977). Acute and chronic hepatitis revisited. Lancet, ii, 914-918.

RIGGS, B.L., SEEMAN, E., HODGSON, S.F. \& TARES, D.
(1981). The fluoride/calcium regimen for primary osteoporosis. In Osteoporosis: Recent Advances in Pat $\$$ ogenesis and Treatment. De Luca, H.F., Frost, H.M., Jes. W.S., Johnston, C. \& Parfitt, A.M. (eds). p. 331. Univer: sity Park Press: Baltimore.

STELLON, A., DAVIES, A., COMPSTON, J.E. \& WILLIAMS R.(1984). Bone loss in corticosteroid treated patients wiț HBsAg-negative chronic active hepatitis. Gut, 25, A558 $\overline{\bar{\alpha}}$ VEDI, S., COMPSTON, J.E., WEBB, A. \& TIGHE, J.R. (1982, Histomorphometric analysis of bone biopsies from the iliac crest of normal British subjects. Metabolic Bono Disease and Related Research, 4, 231.

WINDSOR, A.C.M., MISRA, D.P., LOUDON, J.M. \& STADDON, G.E. (1973). The effect of whole bone extract on ${ }^{47} C_{\overparen{d}}$ absorption in the elderly. Age and Aging, 2, 230.

WOOTON, R. \& REEVE, J. (1980). The relative merits various techniques for measuring radio-calcium absorp tion. Clinical Science, 58, 287. 